\title{
Simultaneous Flow of Two Immiscible Fractional Maxwell Fluids with the Clear Region and Homogeneous Porous Medium
}

(Aliran Serentak bagi Dua Bendalir Maxwell Pecahan tak Tercampur dengan Rantau Jernih dan Medium Berliang Homogen)

\author{
Abdul RauF*, QAmmar Rubbab, Dumitru Vieru \& Ali Majeed
}

\begin{abstract}
One-dimensional transient flows of two layers immiscible fractional Maxwell fluids in a rectangular channel is investigated. The studied problem is based on a mathematical model focused on the fluids with memory described by a constitutive equation with time-fractional Caputo derivative. The flow domain is considered two regions namely one clear region and another filled with a homogeneous porous medium saturated by a generalized Maxwell fluid. Semianalytical and analytical solutions to the problem with initial-boundary conditions and interface fluid-fluid conditions are determined by employing the integral transform method (the Laplace transform and the finite sine-Fourier transform). Talbot's algorithm for the numerical inversion of the Laplace transforms is employed. The memory effects and the influence of the porosity coefficient on the fluid motion are studied. Numerical results and graphical illustrations obtained using the Mathcad software are utilised to analyze the fluid behavior. The influence of the memory on the fluid motion is significant at the beginning of motion and it is attenuated as time passes by.
\end{abstract}

Keywords: Analytical and semi-analytical solutions; fractional Maxwell fluids; memory effects; simultaneous clear and porous medium; two-layered immiscible fluids

\section{ABSTRAK}

Aliran sementara satu dimensi bagi dua lapisan bendalir Maxwell pecahan yang tidak tercampur dalam saluran segi empat dikaji. Masalah yang dikaji berdasarkan model matematik yang berfokus pada bendalir dengan memori yang diperihalkan oleh persamaan juzuk dengan terbitan Caputo pecahan masa. Domain aliran dianggap dua rantau iaitu satu rantau jernih dan satu lagi diisi dengan medium berliang homogen yang tepu oleh bendalir Maxwell teritlak. Penyelesaian semi-analitik dan analitis untuk masalah dengan keadaan sempadan awal dan keadaan antara muka bendalir ditentukan dengan menggunakan kaedah penjelmaan kamiran (jelmaan Laplace dan jelmaan sinus-Fourier terhingga). Algoritma Talbot untuk songsangan berangka bagi jelmaan 'Laplace' digunakan. Kesan memori dan pengaruh pekali keliangan pada pergerakan bendalir dikaji. Hasil berangka dan ilustrasi grafik yang diperoleh menggunakan perisian Mathcad digunakan untuk menganalisis telatah bendalir. Pengaruh memori pada gerakan bendalir adalah signifikan pada awal gerakan dan ia dilemahkan apabila masa berlalu.

Kata kunci: Bendalir Maxwell pecahan; dua lapisan bendalir tak tercampur; kesan memori; penyelesaian analitik dan semi-analitik; serentak jernih dan medium berliang

\section{INTRODUCTION}

The study of simultaneous flow of two or more immiscible fluids in porous as well as in clear medium is significant due to its wide applications in science, medical, geophysics, industry, petroleum engineering, and hydrogeology (Bear 2013; Dullien 2012; Lake 1989; Satpathi et al. 2003). Various applications include oil recovery, blood flow through capillary vessels, equipment cleaning, biofilms, and mucus flow in living cells, removal of carbon dioxide from the atmosphere, groundwater management, crude oil flow through pipelines, bubble generation in microfluidics and bubble trains flow in various complex porous systems. Several researchers have studied the stability/instability of two-layer or multi-layer immiscible fluids flow (Gin \& Daripa 2015; Papaefthymiou \& Papageorgiou 2017; Ward et al. 2019). The linear stability of the viscoelastic two- 
layered plane Poiseuille and Couette flows have been first studied by Yih (1967) with the help of long-wave approach. He observed that both density and viscosity stratification can cause interfacial Kelvin-Helmholtz instability. Herve Le Meur (1997) has studied the uniqueness and the existence of the multi-layered Poiseuille/Couette fluid flow in pipes/channel and observed that interpolated Oldroyd derivative parameter and the viscosity ratios are significant for a unique solution. Kalogirou and Blyth (2019) have considered the Couette-Poiseuille flow of the two-layered superposed fluids to discuss stability. The fluid at the lower layer is populated with surfactants and these surfactants get adsorbed on the interface. It has been observed that if the thickness ratio is much higher than the fluid viscosity ratio and if the surfactant is sufficiently soluble, the flow is stable.

Kim et al. (2019) have worked on the two-layered immiscible Couette flow with the help of a hybrid method. The flow is between two parallel planes in which the upper plane is moving while the lower plane is kept stationary. It has been found that the viscosity ratio has strong effects on the fluid velocity than the surface energy. Khan et al. (2016) have investigated the heat transfer and the fluid velocity of the two-layer immiscible fluid in the presence of pressure type die. The first layer is filled with the inelastic fluid, namely, power-law fluid and the second layer are filled with the viscoelastic liquid (Phan-ThienTanner fluids). It has been seen that the fluid velocity and the fluid temperature increase with the increase in the Deborah number. Other interesting results relating to simultaneous flow two or more fluids can be seen in Aliyu et al. (2017), Ashraf and Phirani (2019), Barannyk et al. (2015), Funahashi et al. (2018), Hisham et al. (2018), Joseph and Renardy (1995), Papaefthymiou et al. (2017) and Rauf et al. (2019).

The study of the viscoelastic fluid flows in the porous medium is of great interest for many industrial and engineering complex processes such as manufacturing processes of the composite materials, paper and textile coating, and optimization of the oil recovery. In Hansen et al. (2018), authors have worked on incompressible and simultaneous two-layered flow in a porous medium and have derived a set of equations which relates the seepage velocities of the fluid components on continuum level where differentiation make sense. The enhancement of thermal transport in Marangoni convective flows of a water-based hybrid nanofluid over a stretched/shrinked sheet has been investigated by Khashi'Ie et al. (2020). In their study, authors considered the presence of surface tension generated by the temperature gradient at the wall surface. Using software MATLAB, authors have determined dual numerical solutions for velocity and temperature. The influence of system parameters on the heat transfer and fluid motion was analyzed.
Modelling of complex systems with the fractional order differential and integral operators have applications in many fields of science such as geophysics, biology, demography, bioengineering, physics, and mathematics (Caputo \& Fabrizio 2015). There exist many fractional differential operators in literature such as Caputo-Fabrizio fractional derivative (Caputo \& Fabrizio 2015), RiemannLiouvillefractional integral/derivative (Podlubny 1999), Caputo fractional derivative (Caputo 1967) and YangSrivastava-Machado fractional derivative (Xiao-Jun 2016), are some of the examples of the fractional-order differential operators used in viscoelasticity, mass and heat transport processes. Hristov (2017) studied the transient space-fractional diffusion with power-law super diffusivity modelled by the Riemann-Liouville fractional derivative. Liu and Pan (2019) have developed a numerical algoritm for a class of fractional differential equations with variable order. Authors used the Legendre polynomial method for the startining solutions of the studied equations. In this paper, we have studied the simultaneous flow of the two immiscible fractional Maxwell fluids between two parallel plates. We have divided the region between two parallel plates into two simultaneous layers. The upper layer is porous whereas the lower layer is clear with no porosity effect. We have considered an unsteady, incompressible and one dimensional fully developed flow which is generated by the movement of the boundary walls and the time-dependent pressure gradient within the fluid layers.

Moreover, we have considered the linear interfacial fluid-fluid condition between two consecutive layers. To find analytical solutions for velocities we have used finite Fourier sine transform in conjunction with the Laplace transformation. A semi-analytical solution for velocity field is recovered with the help of Laplace transform and Talbot's algorithms used for the numerical Laplace inversion.

\section{MATERIALS AND MeTHods}

\section{BASIC CONSTITUTIVE EQUATIONS}

The constitutive equation of the upper convected Maxwell fluid is (Malek et al. 1993).

$$
-p \mathbf{I}+\sigma=\tau,
$$

where $p$ represents the pressure; $\tau$ represents the Cauchy stress tensor; I represents the unit tensor; and $\sigma$ is the extra-stress tensor given by the equation

$$
\begin{aligned}
& \sigma+\lambda \hat{\sigma}=\mu A, A=\nabla \mathbf{v}+(\nabla \mathbf{v})^{T} \\
& \hat{\sigma}=\frac{\partial \sigma}{\partial t}+\mathbf{v} \cdot \nabla \sigma-(\nabla \mathbf{v})^{T} \sigma-\sigma(\nabla \mathbf{v}) .
\end{aligned}
$$


In (2), $\mathbf{v}, \lambda, \mu, \nabla \mathbf{v}$, and the $T$ index are the velocity field, the relaxation time, the fluid viscosity, the velocity gradient tensor, and the transposition operation, respectively.

For the Maxwell fluid upper convected model, the generalized constitutive equation with time-fractional Caputo derivative is (Friedrich 1991; Hristov 2019)

$\sigma+\lambda^{\alpha c} D_{t}^{\alpha} \sigma+\lambda\left[\mathbf{v} \cdot \nabla \sigma-(\nabla \mathbf{v})^{T} \sigma-\sigma(\nabla \mathbf{v})\right]=\mu A, 0<\alpha \leq 1$,

where the Caputo derivative operator ${ }^{c} D_{t}^{\alpha} \sigma(x, y, z, t), 0<\alpha \leq 1$ is defined as (Caputo 1967)

$$
\begin{aligned}
& { }^{c} D_{t}^{\alpha} \sigma= \begin{cases}\frac{\dot{\sigma}^{*} t^{-\alpha}}{\Gamma(1-\alpha)} & \alpha \in(0,1) \\
\dot{\sigma}^{*} \delta(t) & \alpha=1\end{cases} \\
& \dot{\sigma}=\frac{\partial \sigma}{\partial t},
\end{aligned}
$$

$\delta$-being Dirac's distribution.

It is clear from (4) and (5) that, for $\alpha=1$, the fractional constitutive (3) leads to the classical constitutive (2) and for $\lambda=0$, (3) leads to the Newtonian fluid. For the onedimensional flow with the extra-stress tensor and the velocity field given by

$$
\sigma=\sigma(y, t), \sigma(y, 0)=0, \mathbf{v}(x, y, z, t)=u(y, t) \mathbf{e}_{x}, u(y, 0)=0,
$$

where $\mathbf{e}_{x}$ represents the unit vector along the $\mathrm{x}$-axis in the Cartesian coordinate system $O x y z$, we get

$$
\begin{gathered}
\sigma_{x z}=\sigma_{y y}=\sigma_{y z}=\sigma_{z z}=0, \\
\left(1+\lambda^{\alpha c} D_{t}^{\alpha}\right) \sigma_{x y}=\mu \frac{\partial u}{\partial y}, \\
\left(1+\lambda^{\alpha c} D_{t}^{\alpha}\right) \sigma_{x x}=2 \lambda \sigma_{x y} \frac{\partial u}{\partial y} .
\end{gathered}
$$

Let us consider a porous medium of permeability $K$ saturated by a Maxwell fluid. By analogy with the constitutive (8), we can write the following filtration law as (Alishaev \& Mirzadjanzade 1975; Khuzhayorov et al. 2000; Nield 2000)

$$
u=-\frac{K}{\mu \varphi}\left(1+\lambda \frac{\partial}{\partial t}\right) \frac{\partial p}{\partial x} .
$$

Into (10), the velocity $u$, averaged over the pore space, is related to Darcy's velocity by

$$
u_{D}=\varphi u
$$

where $\varphi$ represents the porosity effects of the porous medium. In this work, we will consider a generalized form of the filtration law (10), namely (Govindarajan 2004)

$$
\left(1+\lambda^{\alpha{ }^{c}} D_{t}^{\alpha}\right) \frac{\partial p}{\partial x}=-\frac{\mu \varphi}{K} u .
$$

Generally, the fractional parameters corresponding to the shear stress, respectively to the filtration law are different. To simplify calculations, in this paper we considered these parameters equal.

The local volume-average balance of linear momentum is given by (Vafai \& Tien 1981; Xue \& Nie 2008)

$$
\frac{\partial u}{\partial t}+\frac{1}{\rho} \frac{\partial p}{\partial x}=\frac{1}{\rho} \frac{\partial \tau_{x y}}{\partial y}+\frac{r}{\rho}
$$

where $r$ represents the Darcy's resistance.

It has been observed that the pressure gradient $\frac{\partial p}{\partial x}$ given by (12) can be considered as a measure of the same flow resistance in the porous medium bulk (Khuzhayorov et al. 2000). Moreover, Darcy's resistance $r$ in (13) is as well in effect a flow resistance measure caused by the solid matrix, from (12) we have

$$
r+\lambda^{\alpha{ }^{c}} D_{t}^{\alpha} r=-\frac{\mu \varphi}{K} u
$$

Neglecting the pressure gradient $\frac{\partial p}{\partial x}$ in the direction of the flow, (13) and (14) lead to the following equation of the linear momentum:

$$
\left(1+\lambda^{\alpha c} D_{t}^{\alpha}\right) \frac{\partial u}{\partial t}=v \frac{\partial^{2} u}{\partial y^{2}}-\frac{\mu \varphi}{K} u .
$$

For a clear fluid $(\mathrm{r}=0)$ and zero pressure gradient $\frac{\partial p}{\partial x}$ in the direction of the flow, (14) leads to the flow equation

$$
\left(1+\lambda^{\alpha c} D_{t}^{\alpha}\right) \frac{\partial u}{\partial t}=v \frac{\partial^{2} u}{\partial y^{2}} .
$$

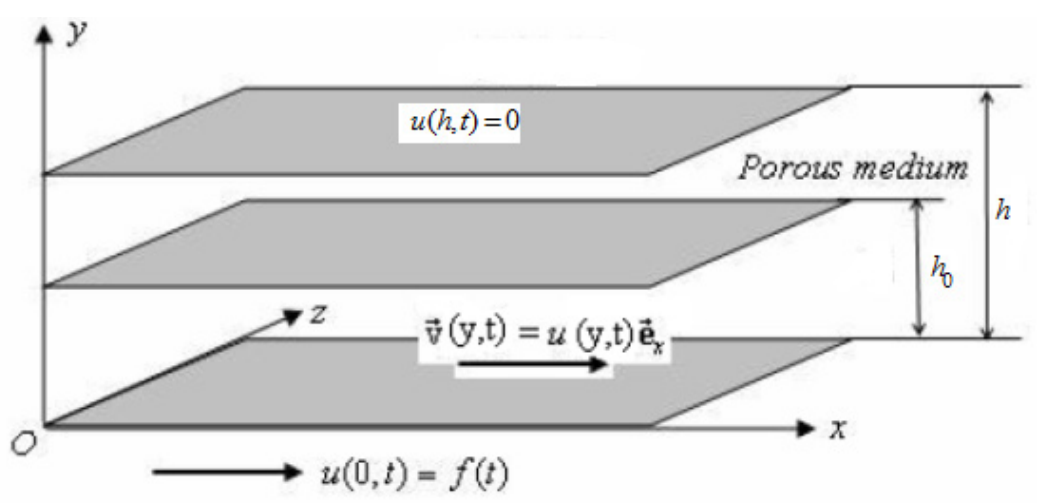

FIGURE 1. Flow geometry 


\section{MATHEMATICAL MODELING}

Let us consider the flow of an upper convected Maxwell fluid with generalized constitutive equation between two horizontal parallel impermeable plates situated in the plane $y=0$, respectively $y=h$ of the coordinate system $O x y z$. The clear generalized Maxwell fluid flows in the layer $0 \leq y \leq h_{0} \leq h$. A porous layer of thickness $h-h_{0}$ is attached to the upper plate and is saturated by a generalized Maxwell fluid (Figure 1 with non-dimensional parameters). At the instant $t=0$, both plates and the fluid are at rest. In this work, we investigate flows with the velocity field $\mathbf{v}(y, t)=u(y, t) \mathbf{e}_{x}$ generated by the motion of the plate $y=0$. The governing equations of the fluid flow are given by Xue and Nie (2008)

For clear fluid region, $y \in\left[0, h_{0}\right]$

$$
\left(1+\lambda^{\alpha c} D_{t}^{\alpha}\right) \frac{\partial u(y, t)}{\partial t}=v \frac{\partial^{2} u}{\partial y^{2}},
$$

For porous medium region, $y \in\left[h_{0}, h\right]$

$$
\left(1+\lambda^{\alpha c} D_{t}^{\alpha}\right) \frac{\partial v(y, t)}{\partial t}+\frac{\mu \varphi}{K} v(y, t)=v \frac{\partial^{2} v}{\partial y^{2}} .
$$

Along with (17) and (18), the following initial and boundary conditions are considered:

$$
\begin{gathered}
v(y, 0)=\left.\frac{\partial v(y, t)}{\partial t}\right|_{t=0}=0, u(y, 0)=\left.\frac{\partial u(y, t)}{\partial t}\right|_{t=0}=0, \\
\mathrm{u}(0, \mathrm{t})=\mathrm{U}_{0} \mathrm{f}_{1}(\mathrm{t}), \mathrm{U}_{0}>0, \mathrm{v}(\mathrm{h}, \mathrm{t})=0, \\
v\left(h_{0}, t\right)=u\left(h_{0}, t\right),\left.\frac{\partial v(y, t)}{\partial y}\right|_{y=h_{0}}=\left.\frac{\partial u(y, t)}{\partial y}\right|_{y=h_{0}} .
\end{gathered}
$$

Using the following dimensionless quantities

$$
\begin{aligned}
& u^{*}=\frac{u}{U_{0}}, v^{*}=\frac{v}{U_{0}}, y^{*}=\frac{U_{0}}{v} y, t^{*}=\frac{U_{0}^{2}}{v} t, \lambda^{* \alpha}=\left(\frac{\lambda U_{0}^{2}}{v}\right)^{\alpha}, d_{0}=\frac{h_{0} U_{0}}{v}, \\
& \gamma=\frac{v^{2} \varphi}{K U_{0}^{2}}, d=\frac{U_{0} h}{v}, f^{*}\left(t^{*}\right)=f_{1}\left(\frac{v}{U_{0}^{2}} t\right),
\end{aligned}
$$

Equations (17)-(21) become (neglecting the notation " **")

$$
\begin{gathered}
\left(1+\lambda^{\alpha}{ }^{c} D_{t}^{\alpha}\right) \frac{\partial u(y, t)}{\partial t}=\frac{\partial^{2} u}{\partial y^{2}}, \quad y \in\left[0, d_{0}\right], \\
\left(1+\lambda^{\alpha}{ }^{c} D_{t}^{\alpha}\right) \frac{\partial v(y, t)}{\partial t}=\frac{\partial^{2} v}{\partial y^{2}}-\gamma v(y, t), y \in\left[d_{0}, d\right], \\
u(y, 0)=\left.\frac{\partial u(y, t)}{\partial t}\right|_{t=0}=0, v(y, 0)=\left.\frac{\partial v(y, t)}{\partial t}\right|_{t=0}=0, \\
\mathrm{u}(0, \mathrm{t})=\mathrm{f}(\mathrm{t}), \mathrm{v}(\mathrm{d}, \mathrm{t})=0, \\
v\left(d_{0}, t\right)=u\left(d_{0}, t\right),\left.\frac{\partial v(y, t)}{\partial y}\right|_{y=d_{0}}=\left.\frac{\partial u(y, t)}{\partial y}\right|_{y=d_{0}}
\end{gathered}
$$

\section{SOLUTION OF THE PROBLEM}

Taking the Laplace transform to (23), (24), (26), (27) and using the initial conditions (25), we get

$$
\begin{gathered}
\frac{\partial^{2} \bar{u}(y, s)}{\partial y^{2}}=s\left(\lambda^{\alpha} s^{\alpha}+1\right) \bar{u}(y, s), y \in\left[0, d_{0}\right], \\
\frac{\partial^{2} \bar{v}(y, s)}{\partial y^{2}}=\left(s\left(\lambda^{\alpha} s^{\alpha}+1\right)+\gamma\right) \bar{v}(y, s), y \in\left[d_{0}, d\right], \\
\bar{u}(0, s)=\bar{f}(s), \bar{v}(d, s)=0, \\
\bar{v}\left(d_{0}, s\right)=\bar{u}\left(d_{0}, s\right),\left.\frac{\partial \bar{v}(y, s)}{\partial y}\right|_{y=d_{0}}=\left.\frac{\partial \bar{u}(y, s)}{\partial y}\right|_{y=d_{0}}
\end{gathered}
$$

where $\bar{G}(y, s)=\int_{0}^{\infty} G(y, t) e^{-s t} d t$ denotes the Laplace transform of the function $G(y, t)$.

\section{SEMI-ANALYTICAL SOLUTION}

The generic solution of (28) and (29) are

$$
\bar{u}(y, s)=A_{1}(s) \exp \left(-y \sqrt{w_{1}(s, \alpha)}\right)+A_{2}(s) \exp \left(y \sqrt{w_{1}(s, \alpha)}\right),
$$

$$
\bar{v}(y, s)=A_{3}(s) \exp \left(-y \sqrt{w_{2}(s, \alpha)}\right)+A_{4}(s) \exp \left(y \sqrt{w_{2}(s, \alpha)}\right),
$$

where

$$
w_{1}(s, \alpha)=s\left(1+\lambda^{\alpha} s^{\alpha}\right), w_{2}(s, \alpha)=s\left(1+\lambda^{\alpha} s^{\alpha}\right)+\gamma .
$$

Using the boundary and interface fluid-solid matrix conditions (30) and (31), we obtain that parameters $A_{i}(s), i=1,2,3,4$, are given by

$A_{1}(s)=\frac{f(s) e^{d_{0} \sqrt{w_{1}(s)}}\left(\sqrt{w_{2}(s)} \cosh \left(\left(d-d_{0}\right) \sqrt{w_{2}(s)}\right)+\sqrt{w_{1}(s)} \sinh \left(\left(d-d_{0}\right) \sqrt{w_{2}(s)}\right)\right)}{D(s)}$,

$A_{2}(s)=-\frac{f(s) e^{-d_{0} \sqrt{w_{1}(s)}}\left(\sqrt{w_{2}(s)} \cosh \left(\left(d-d_{0}\right) \sqrt{w_{2}(s)}\right)-\sqrt{w_{1}(s)} \sinh \left(\left(d-d_{0}\right) \sqrt{w_{2}(s)}\right)\right)}{D(s)}$,

$$
\begin{aligned}
& A_{3}(s)=\frac{f(s) \sqrt{w_{1}(s)} e^{d \sqrt{w_{2}(s)}}}{D(s)}, \\
& A_{4}(s)=\frac{-f(s) \sqrt{w_{1}(s)} e^{-d \sqrt{w_{2}(s)}}}{D(s)},
\end{aligned}
$$

$D(s)=\sqrt{w_{1}(s)} D_{1}(s)+\sqrt{w_{2}(s)} D_{2}(s)$,

$D_{1}(s)=2 \cosh \left(d_{0} \sqrt{w_{1}(s)}\right) \sinh \left(\left(d-d_{0}\right) \sqrt{w_{2}(s)}\right)$,

$D_{2}(s)=2 \sinh \left(d_{0} \sqrt{w_{1}(s)}\right) \cosh \left(\left(d-d_{0}\right) \sqrt{w_{2}(s)}\right)$. 
It is not easy to apply the analytic method to compute the inverse Laplace transform of (32)-(35), since complicated expressions are involved in these equations. To overcome this issue, we have used two accuracy numerical algorithms, namely the improved Talbot algorithm (Dingfelder \& Weideman 2015) and the fixed Talbot algorithm (Abate \& Valko 2004) for the computation of the numerical inversion of Laplace transform.

Let $\bar{G}(y, s)=\int_{0}^{\infty} G(y, t) e^{-s t} d t$. The Talbot algorithm (Bracewell 2000) for the Laplace transform inversion of the function $\bar{G}(y, s)$ is

$$
\begin{aligned}
G(y, t) \cong & \frac{r_{1}}{M}\left\{\sum_{k=1}^{M-1} \operatorname{Re}\left[\exp \left(t z\left(\theta_{k}\right)\right) \bar{G}\left(y, z\left(\theta_{k}\right)\right)\left(1+i \zeta\left(\theta_{k}\right)\right)\right]\right. \\
& \left.+\frac{\exp \left(r_{1} t\right)}{2} \bar{G}\left(y, r_{1}\right)\right\},
\end{aligned}
$$

where

$$
\begin{aligned}
& r_{1}=\frac{2 M}{5 t}, z(\theta)=r_{1} \theta i+r_{1} \theta \cot \theta,-\pi<\theta<\pi, \\
& \zeta(\theta)=(\theta \cot \theta-1) \cot \theta+\theta, \theta_{k}=\frac{k \pi}{M} .
\end{aligned}
$$

Another method to approximate the function $G(y, t)$ is the improved Talbot algorithm for the inverse Laplace transform and is given by (Lorenzo \& Hartley 1999)

$$
G(y, t) \cong \sum_{k=1}^{M} \frac{\bar{G}\left(y, z_{1}\left(\sigma_{k}\right)\right)\left(v+i \zeta_{1}\left(\sigma_{k}\right)\right) \exp \left(t z_{1}\left(\sigma_{k}\right)\right)}{t},
$$

where

$$
\begin{aligned}
& z_{1}(\theta)=\frac{M}{t}[v i \theta+\mu \theta \cot (\alpha \theta)-\xi],-\pi \leq \theta \leq \pi \\
& \zeta_{1}(\theta)=\alpha \mu \theta+\mu(\alpha \theta \cot (\alpha \theta)-1) \cot (\alpha \theta), \sigma_{k}=\frac{(2 k-1) \pi}{M}-\pi .
\end{aligned}
$$

Here $\alpha, M, v, \mu, \xi$ are parameters to be specified by the user.

\section{ANALYTICAL SOLUTION FOR THE VELOCITY}

To find the exact solution of velocities $u(y, t)$ and $v(y, t)$ we will use the finite Fourier sine transform along with the Laplace transform.

The finite Fourier sine transform of the function $\bar{\varphi}(y, s), a \leq y \leq b, a<b$, is defined as (Russell \& Charles 1959)

$$
\tilde{\bar{\varphi}}_{m}(s)=\int_{a}^{b} \bar{\varphi}(y, s) \sin \left(\xi_{m}(y-a)\right) d y, \xi_{m}=\frac{m \pi}{b-a}, m=1,2, \cdots,
$$

along with the inverse Fourier transform defined by

$$
\bar{\varphi}(y, s)=\sum_{m=1}^{\infty} 2 \frac{\tilde{\bar{\varphi}}_{m}(s) \sin \left(\xi_{m}(y-a)\right)}{b-a} .
$$

With the application of finite Fourier sine transform (40) to (28) and (29) along with the boundary conditions (30) and (31), the transformed velocities take the form

$$
\begin{gathered}
\tilde{\bar{u}}_{m}(s)=-\frac{(-1)^{m} \xi_{1 m}}{s\left(1+\lambda^{\alpha} s^{\alpha}\right)+\xi_{1 m}^{2}} \bar{u}\left(d_{0}, s\right)+\frac{\xi_{1 m} \bar{f}(s)}{s\left(1+\lambda^{\alpha} s^{\alpha}\right)+\xi_{1 m}^{2}}, \\
\xi_{1 m}=\frac{m \pi}{d_{0}}, m=1,2, ., \\
\tilde{\bar{v}}_{m}(s)=\frac{\xi_{2 m}}{s\left(1+\lambda^{\alpha} s^{\alpha}\right)+\xi_{2 m}^{2}+\gamma} \bar{v}\left(d_{0}, s\right), \\
\xi_{2 m}=\frac{m \pi}{d-d_{0}}, m=1,2, \cdots .
\end{gathered}
$$

Velocity function (42) can be written in equivalent form as

$$
\begin{aligned}
& \tilde{\bar{u}}_{m}(s)=\frac{1}{\xi_{1 m}} \bar{f}(s)-\frac{(-1)^{m}}{\xi_{1 m}} \bar{u}\left(d_{0}, s\right)+\frac{(-1)^{m} s\left(1+\lambda^{\alpha} s^{\alpha}\right)}{\xi_{1 m}\left(s\left(1+\lambda^{\alpha} s^{\alpha}\right)+\xi_{1 m}^{2}\right)} \bar{u}\left(d_{0}, s\right) \\
& -\frac{s\left(1+\lambda^{\alpha} s^{\alpha}\right) \bar{f}(s)}{\xi_{1 m}\left(s\left(1+\lambda^{\alpha} s^{\alpha}\right)+\xi_{1 m}^{2}\right)}, \quad \xi_{1 m}=\frac{m \pi}{d_{0}}, m=1,2, \cdots .
\end{aligned}
$$

Considering the following pair functions $\psi_{1}(y)$ and $\psi_{2}(y)$ along with their inverse Fourier sine transform

$$
\begin{aligned}
& \psi_{1}(y)=\frac{-y+d_{0}}{d_{0}}, 0 \leq y \leq d_{0}, \tilde{\psi}_{1 m}=\frac{1}{\xi_{1 m}}, \\
& \psi_{2}(y)=\frac{y}{d_{0}}, 0 \leq y \leq d_{0}, \tilde{\psi}_{2 m}=\frac{(-1)^{m+1}}{\xi_{1 m}},
\end{aligned}
$$

and taking the inverse Fourier sine transform to (45) we get

$$
\begin{aligned}
\bar{u}(y, s)= & \frac{y}{d_{0}} \bar{u}\left(d_{0}, s\right)+\frac{d_{0}-y}{d_{0}} \bar{f}(s)+ \\
& \frac{2 \bar{u}\left(d_{0}, s\right)}{d_{0}} \sum_{m=1}^{\infty} \frac{(-1)^{m} s\left(1+\lambda^{\alpha} s^{\alpha}\right)}{\xi_{1 m}\left(s\left(1+\lambda^{\alpha} s^{\alpha}\right)+\xi_{1 m}^{2}\right)} \sin \left(\xi_{1 m} y\right) \\
& -\frac{2 \bar{f}(s)}{d_{0}} \sum_{m=1}^{\infty} \frac{s\left(1+\lambda^{\alpha} s^{\alpha}\right) \sin \left(\xi_{1 m} y\right)}{\xi_{1 m}\left(s\left(1+\lambda^{\alpha} s^{\alpha}\right)+\xi_{1 m}^{2}\right)} .
\end{aligned}
$$

A similar manner leads to

$$
\tilde{\bar{v}}_{m}(s)=\frac{1}{\xi_{2 m}} \bar{v}\left(d_{0}, s\right)-\frac{s\left(1+\lambda^{\alpha} s^{\alpha}\right)}{\xi_{2 m}\left(s\left(1+\lambda^{\alpha} s^{\alpha}\right)+\xi_{2 m}^{2}+\gamma\right)} \bar{v}\left(d_{0}, s\right),
$$

and considering

$$
\psi_{3}(y)=\frac{d-y}{d-d_{0}}, d_{0} \leq y \leq d, \tilde{\psi}_{3 m}=\frac{1}{\xi_{2 m}},
$$

and the implication of the inverse Fourier sine transform to (48) gives

$$
\begin{aligned}
\bar{v}(y, s)=\frac{d-y}{d-d_{0}} \bar{v}\left(d_{0}, s\right)-\frac{2 \bar{v}\left(d_{0}, s\right)}{d-d_{0}} \sum_{m=1}^{\infty} \\
\frac{s\left(1+\lambda^{\alpha} s^{\alpha}\right)}{\xi_{2 m}\left(s\left(1+\lambda^{\alpha} s^{\alpha}\right)+\xi_{2 m}^{2}+\gamma\right)} \sin \left(\xi_{2 m}\left(y-d_{0}\right)\right),
\end{aligned}
$$

Using (21), the Laplace transformed form of the velocities on the interface $y=d_{0}$ are

$$
\bar{v}\left(d_{0}, s\right)=\bar{u}\left(d_{0}, s\right)=\bar{A}(s) \bar{T}_{12}\left(d_{0}, s\right) \bar{f}(s),
$$


where

$A(s)=\frac{1}{\bar{T}_{11}\left(d_{0}, s\right)+\bar{T}_{21}\left(d_{0}, s\right)}$,

$\bar{T}_{11}(y, s)=\frac{1}{d_{0}}\left(2 \sum_{m=1}^{\infty} \frac{(-1)^{m} s\left(1+\lambda^{\alpha} s^{\alpha}\right)}{s\left(1+\lambda^{\alpha} s^{\alpha}\right)+\xi_{1 m}^{2}} \cos \left(\xi_{1 m} y\right)+1\right)$,

$\bar{T}_{21}(y, s)=\frac{1}{\left(d-d_{0}\right)}\left(2 \sum_{m=1}^{\infty} \frac{s\left(1+\lambda^{\alpha} s^{\alpha}\right)}{\left(s\left(1+\lambda^{\alpha} s^{\alpha}\right)+\xi_{2 m}^{2}+\gamma\right)} \cos \left(\xi_{2 m}\left(y-d_{0}\right)\right)+1\right)$,

$\bar{T}_{12}(y, s)=\frac{1}{d_{0}}\left(2 \sum_{m=1}^{\infty} \frac{s\left(1+\lambda^{\alpha} s^{\alpha}\right) \cos \left(\xi_{1 m} y\right)}{s\left(1+\lambda^{\alpha} s^{\alpha}\right)+\xi_{1 m}^{2}}+1\right)$.

For the inverse Laplace transforms of the functions $\bar{u}(y, s), \bar{v}(y, s)$ and $\bar{u}\left(d_{0}, s\right)=\bar{v}\left(d_{0}, s\right)$ we consider the following auxiliary functions,

$\bar{H}_{1 m}(s):=\frac{s\left(1+\lambda^{\alpha} s^{\alpha}\right)}{s\left(1+\lambda^{\alpha} s^{\alpha}\right)+\xi_{1 m}^{2}}$

$$
\begin{gathered}
=1+\sum_{k=1}^{\infty}(-1)^{k}\left(\frac{\xi_{1 m}^{2}}{\lambda_{\alpha}}\right)^{k}\left[\frac{s^{-k}}{\left(s^{\alpha}+\lambda^{-\alpha}\right)^{k}}\right], \\
\bar{H}_{2 m}(s):=\frac{s\left(1+\lambda^{\alpha} s^{\alpha}\right)}{s\left(1+\lambda^{\alpha} s^{\alpha}\right)+\xi_{2 m}^{2}+\gamma} \\
=1+\sum_{k=1}^{\infty}(-1)^{k}\left(\frac{\xi_{2 m}^{2}+\gamma}{\lambda_{\alpha}}\right)^{k}\left[\frac{s^{-k}}{\left(s^{\alpha}+\lambda^{-\alpha}\right)^{k}}\right] .
\end{gathered}
$$

The generalized G-Lorenzo-Hartley function is defined by Lorenzo and Hartley (1999)

$\mathrm{L}^{-1}\left[\frac{s^{\beta}}{\left(s^{\alpha}-\sigma\right)^{\gamma}}\right]=G_{\alpha, \beta, \gamma}(t, \sigma)=\sum_{k=0}^{\infty} \frac{1}{k !} \frac{\Gamma(k+\gamma)}{\Gamma(\gamma) \Gamma((k+\gamma) \alpha-\beta)} \sigma^{k} t^{(k+\gamma) \alpha-\beta-1}$,

$$
\operatorname{Re}(\alpha \gamma-\beta)>0, \operatorname{Re}(s)>0,\left|\frac{\sigma}{s^{\alpha}}\right|<1 .
$$

The inverse Laplace transform of $\bar{H}_{1 m}(s)$ and $\bar{H}_{2 m}(s)$ takes the form

$$
\begin{aligned}
& H_{1 m}(t)=\delta(t)+\sum_{k=1}^{\infty}(-1)^{k}\left(\frac{\xi_{1 m}^{2}}{\lambda^{\alpha}}\right)^{k} G_{\alpha,-k, k}\left(t,-\lambda^{-\alpha}\right), \\
& H_{2 m}(t)=\delta(t)+\sum_{k=1}^{\infty}(-1)^{k} G_{\alpha,-k, k}\left(t,-\lambda^{-\alpha}\right)\left(\frac{\xi_{2 m}^{2}+\gamma}{\lambda^{\alpha}}\right)^{k},
\end{aligned}
$$

where $\delta(t)$ is Dirac delta function.

Using (46), (49), (55) and (56), we can write the expressions for velocities $u(y, t), v(y, t)$ as:

$$
\begin{gathered}
u(y, t)=\frac{d_{0}-y}{d_{0}} f(t)+\frac{y}{d_{0}} u\left(d_{0}, t\right)+ \\
\frac{2}{d_{0}} \sum_{m=1}^{\infty} \frac{(-1)^{m} \sin \left(\xi_{1 m} y\right)}{\xi_{1 m}} H_{1 m}(t)^{*} u\left(d_{0}, t\right) \\
-\frac{2}{d_{0}} \sum_{m=1}^{\infty} \frac{\sin \left(\xi_{1 m} y\right)}{\xi_{1 m}} f(t)^{*} H_{1 m}(t) \\
v(y, t)=\frac{y-d}{d_{0}-d} v\left(d_{0}, t\right)+\frac{2}{d_{0}-d} \sum_{m=1}^{\infty} \frac{\sin \left(\xi_{2 m}\left(y-d_{0}\right)\right)}{\xi_{2 m}} H_{1 m}(t)^{*} v\left(d_{0}, t\right)
\end{gathered}
$$

where $G(t)^{*} K(t)=\int_{0}^{t} G(t-q) K(q) d q$ represents the convolution product of the functions $G(t), K(t)$ and $u\left(d_{0}, t\right)=v\left(d_{0}, t\right)$ is given by

$$
u\left(\mathrm{~d}_{0}, \mathrm{t}\right)=\mathrm{v}\left(\mathrm{d}_{0}, \mathrm{t}\right)=\mathrm{T}_{12}\left(\mathrm{~d}_{0}, \mathrm{t}\right) * \mathrm{f}(\mathrm{t}) * \mathrm{~A}(\mathrm{t}),
$$

where $A(t)$ is an auxiliary function such that $A(t) * T_{11}\left(d_{0}, t\right)+A(t) * T_{21}\left(d_{0}, t\right)=\delta(t)$, and

$$
\begin{aligned}
& T_{11}(y, t)=\frac{1}{d_{0}}\left(\delta(t)+2 \sum_{m=1}^{\infty}(-1)^{m} H_{1 m}(t) \cos \left(\xi_{1 m} y\right)\right), \\
& \bar{T}_{12}(y, s)=\frac{1}{d_{0}}\left(\delta(t)+2 \sum_{m=1}^{\infty} H_{1 m}(t) \cos \left(\xi_{1 m} y\right)\right), \\
& \bar{T}_{21}(y, s)=\frac{1}{\left(d-d_{0}\right)}\left(1+2 \sum_{m=1}^{\infty} H_{2 m}(t) \cos \left(\xi_{2 m}\left(y-d_{0}\right)\right)\right) .
\end{aligned}
$$
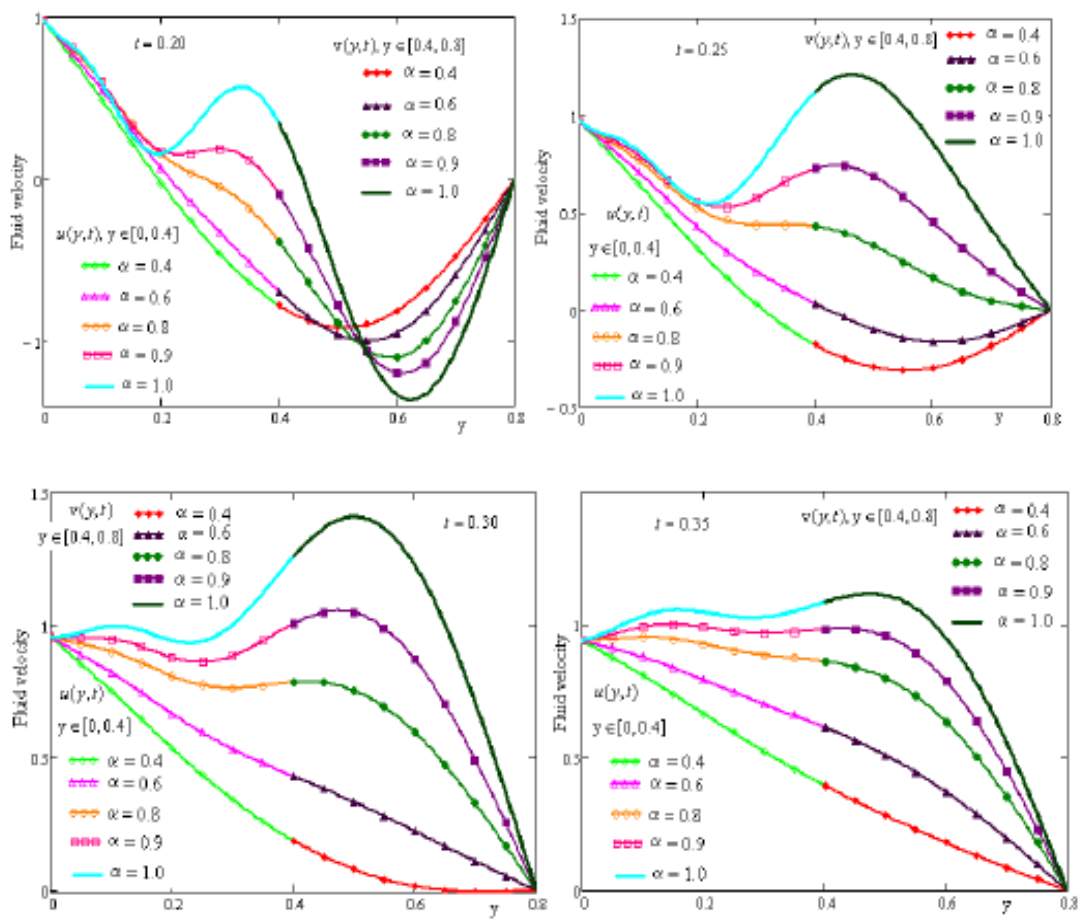

FIGURE 2. The profiles of velocity for small values of the time $t$ and for different values of the fractional parameter $\alpha$ 

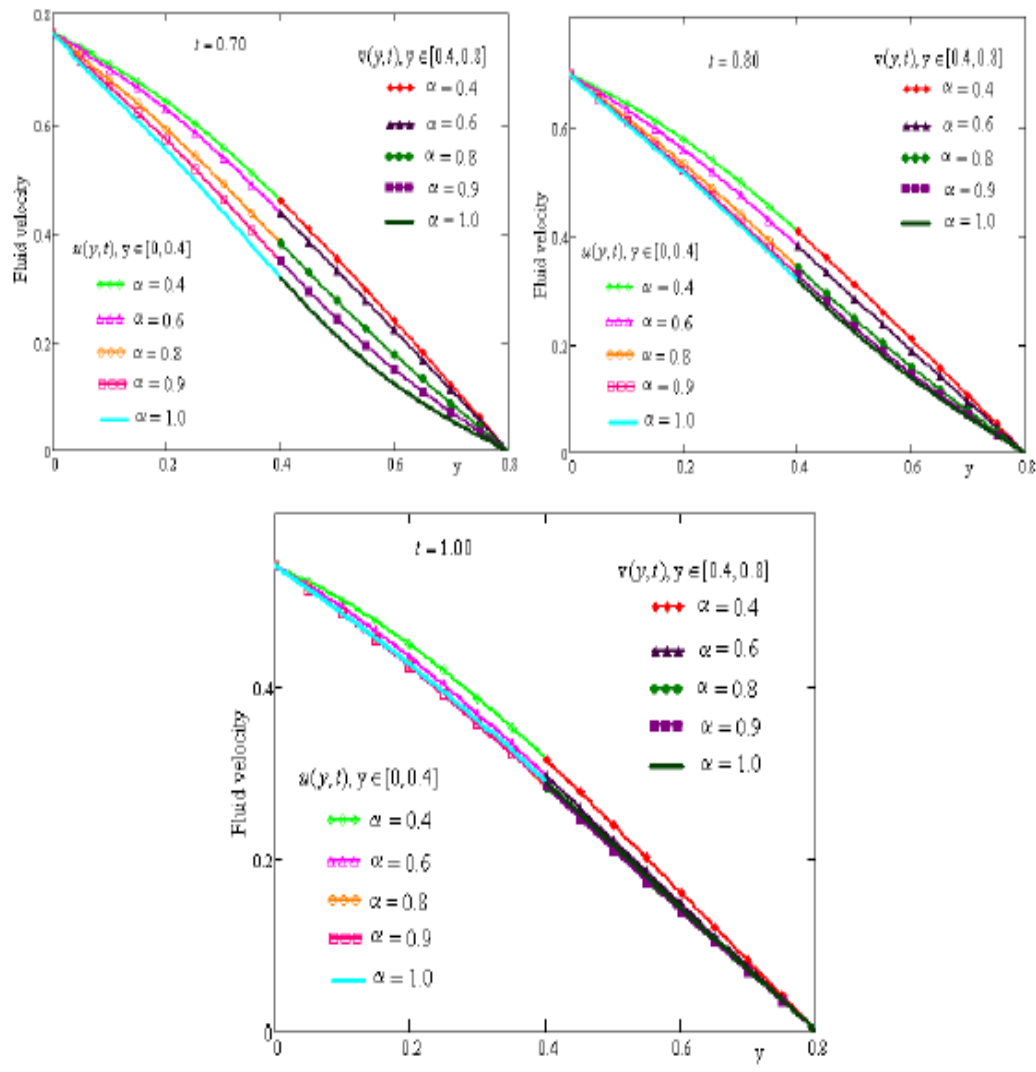

FIGURE 3. The profiles of velocity for large values of the time $t$ and for various values of the fractional parameter $\alpha$
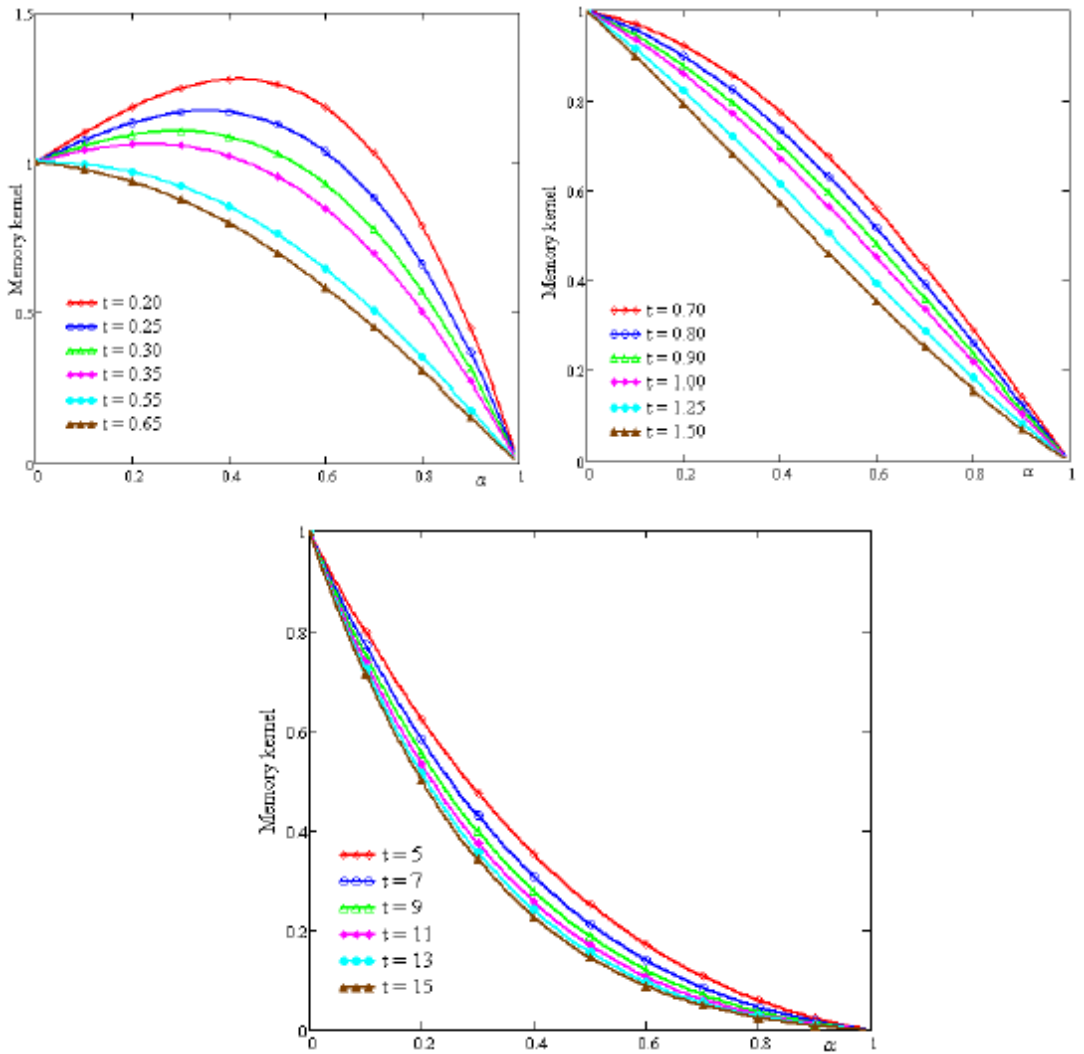
FIGURE 4. The profiles of the kernel $h(\alpha, t)=\frac{t^{-\alpha}}{\Gamma(1-\alpha)}, \alpha \in[0,1]$ for
different values of the time 

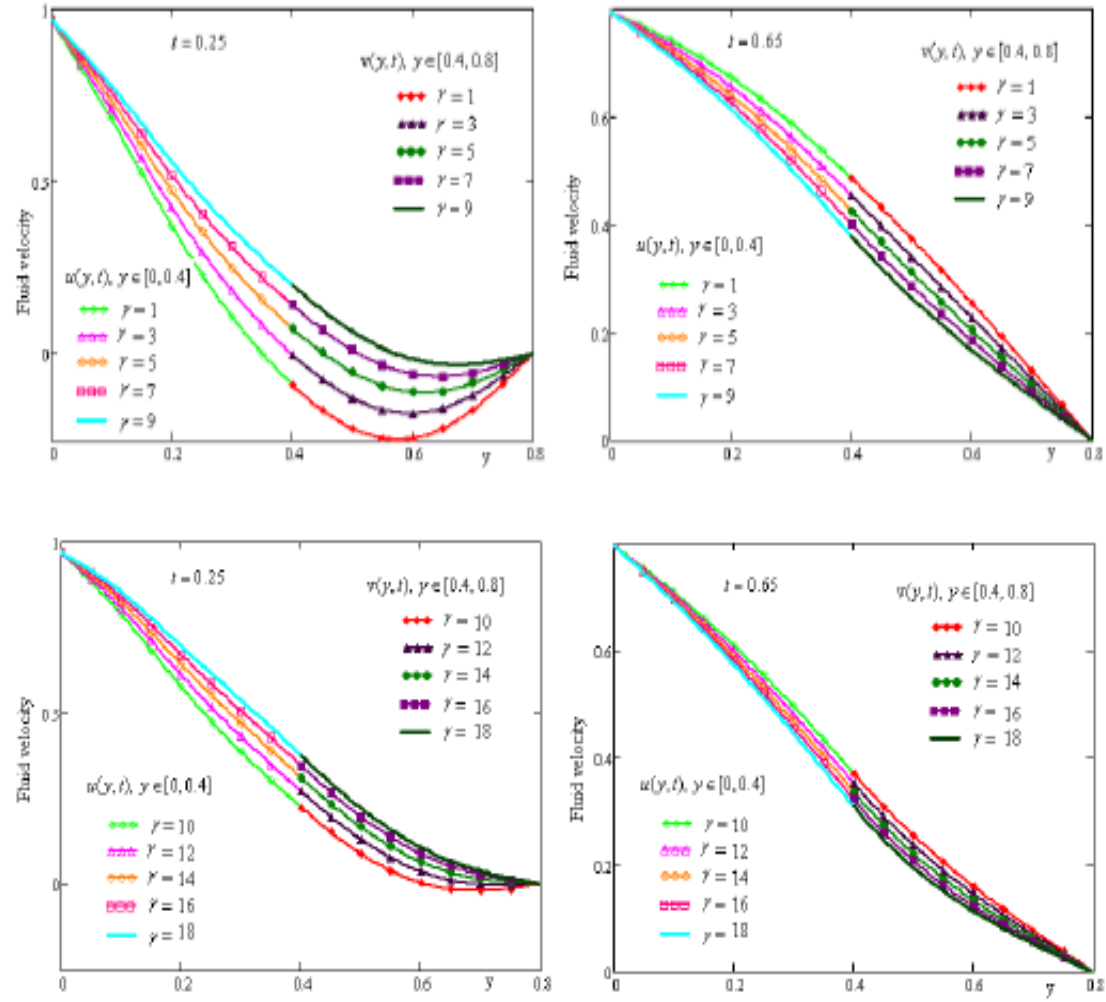

FIGURE 5 . The profiles of velocity for $\alpha=0.5$ and different values of the porosity parameter $\gamma$.

\section{NUMERICAL RESULTS AND DISCUSSION}

Simultaneous one-dimensional flow of two layers unsteady immiscible generalized Maxwell fluids over a rectangular channel have been investigated. In a Cartesian coordinate system, the rectangular channel is determined by two parallel plates situated at $y=0$, respectively, $y=h$. The plane wall at $y=0$ has a translatory motion with velocity $u(0, t)=U_{0} f_{1}(t), U_{0}>0$ being a continuum function of exponential order to infinity and $f_{1}(0)=0$. The plate at plane $y=h$ is at rest.

In this problem, the generalization consists into consideration of the fractional constitutive equation of Maxwell fluids based on Caputo time-fractional derivative.

It is clear from the constitutive (8) that the shear stress $\sigma_{x y}$ is given by

$$
\sigma_{x y}(y, t)=\int_{0}^{t} \frac{\mu}{\lambda^{\alpha}}(t-\eta)^{\alpha-1} E_{\alpha, \alpha}\left(-\lambda^{-\alpha}(t-\eta)^{\alpha}\right) \frac{\partial u(y, \eta)}{\partial \eta} d \eta,
$$

therefore, the history of the velocity gradient influences the shear stress. Such type of flow is the so-called flow with memory.

In the flow channel have been considered two regions, namely the clean region $y \in\left[0, d_{0}\right]$, in which flows a fractional Maxwell fluid and the region $y \in\left[d_{0}, d\right]$, filled with a porous medium saturated by another fractional Maxwell fluid. On the solid boundaries, the no-slip condition is considered, while at the fluid-fluid interface $y=d_{0}$, the velocity and shear stress are considered continuous.

Semi-analytical solutions of the problem with initial, boundary and interface conditions have been determined by employing the Laplace transform along with the Talbot algorithms for the numerical inverse Laplace transforms. Using finite sine-Fourier transform and Laplace transform, the analytical solutions of the same problem have been determined.

To study the influence of shear stress dumping and the porous medium on the fluid velocity we have analyzed the particular case in which the motion of the bottom plate is an oscillation motion with the dimensionless velocity $u(0, t)=\cos (t)-1$. The flows regions are determined by $d_{0}=0.4$ and $d=0.8$.

The dimensional parameters used in this study are $h_{0}=0.05(\mathrm{~m}), h=0.025(\mathrm{~m}), U_{0}=0.008(\mathrm{~m} / \mathrm{s}), \lambda=0.5, v=0.0005$. Numerical results presented in Figures 2 to 5 were obtained 
with the Mathcad software. Figures 2 and 3 illustrate the graphs for the velocity profiles versus the spatial coordinate $y$ at different time instant and for various values of fractional parameter $\alpha$.

As expected, the fluid behavior is significantly different for small values of the time $t$, respectively for large values of the time $t$. This fact is due to the variation in time of the Caputo kernel which is presented in Figure 4. It is observed from Figure 4 that the Caputo kernel increases with the fractional parameter and small values of the time $t$. The kernel of the fractional derivative attains a maximum value for $0.4<\alpha<0.5$. For large values of the time $t$, the derivative kernel decreases with $\alpha$; for values close to 1 , the values of a kernel are very small, therefore, the damping of the velocity gradient will be weaker.

Figure 2 plotted the profiles of velocity for small values of the time $t$ and different values of the fractional parameter, namely $\alpha \in\{0.4,0.6,0.8,0.9,1.0\}$. It is seen from this figure that Maxwell fluids with generalized fractional constitutive equation have a slower motion compared to the ordinary Maxwell fluids corresponding to $\alpha=1$. This behavior is due to the new relationship between shear stress and the shear rate in which the velocity gradient is damped by the Caputo kernel. As it is observed in Figure 3 , the velocity of fluid is not significantly influenced by the memory effects at large values of the time $t$. According with the variation of the derivative kernel (Figure 4), the damping of the velocity gradient is much lower for large values of the time $t$; therefore, the influence of the fractional parameter on the fluid velocity is almost insignificant.

The influence of the non-dimensional porosity parameter $\gamma$ on the fluid velocity is presented in Figure 5 , for the fractional parameter $\alpha=0.5$ and the time $t \in\{0.25,0.65\}$ At small values of the time $t$, the velocity of fractional fluids is increasing with the porosity parameter, while, at large values of the time $t$ the fluid velocity is decreasing with the porosity parameter.

\section{REFERENCES}

Abate, J. \& Valkó, P.P. 2004. Multi-precision Laplace transform inversion. International Journal for Numerical Methods in Engineering 60(5): 979-993.

Alishaev, M.G. \& Mirzadjanzade, A.K. 1975. For the calculation of delay phenomenon in filtration theory. Izvestiya Vysshikh Uchebnykh Zavedeniy. Neft'i Gaz 6: 71-78.

Aliyu, A.M., Baba, Y.D., Lao, L., Yeung, H. \& Kim, K.C. 2017. Interfacial friction in upward annular gas-liquid two-phase flow in pipes. Experimental Thermal and Fluid Science 84(2017): 90-109.

Ashraf, S. \& Phirani, J. 2019. Capillary displacement of viscous liquids in a multi-layered porous medium. Soft Matter 15(9): 2057-2070.

Barannyk, L.L., Papageorgiou, D.T., Petropoulos, P.G. \& VandenBroeck, J.M. 2015. Nonlinear dynamics and wall touch-up in unstably stratified multilayer flows in horizontal channels under the action of electric fields. SIAM Journal on Applied Mathematics 75(1): 92-113.

Bear, J. 2013. Dynamics of Fluids in Porous Media. New York: Courier Corporation. pp. 1-1757.

Bracewell, R.N. \& Bracewell, R.N. 1986. The Fourier Transform and Its Applications (Vol. 31999). New York: McGraw-Hill. pp. 1-368.

Caputo, M. 1967. Linear models of dissipation whose Q is almost frequency independent II. Geophysical Journal International 13(5): 529-539.

Caputo, M. \& Fabrizio, M. 2015. A new definition of fractional derivative without singular kernel. Progress in Fractional Differentiation and Application 1(2): 1-13.

Dingfelder, B. \& Weideman, J.A.C. 2015. An improved Talbot method for numerical Laplace transform inversion. Numerical Algorithms 68(1): 167-183.

Dullien, F.A. 2012. Porous Media: Fluid Transport and Pore Structure. London: Academic Press. pp. 1-567.

Friedrich, C.H.R. 1991. Relaxation and retardation functions of the Maxwell model with fractional derivatives. Rheologica Acta 30(2): 151-158.

Funahashi, H., Kirkland, K.V., Hayashi, K., Hosokawa, S. \& Tomiyama, A. 2018. Interfacial and wall friction factors of swirling annular flow in a vertical pipe. Nuclear Engineering and Design 330: 97-105.

Gin, C. \& Daripa, P. 2015. Stability results for multi-layer radial Hele-Shaw and porous media flows. Physics of Fluids 27(1): 012101.

Govindarajan, R. 2004. Effect of miscibility on the linear instability of two-fluid channel flow. International Journal of Multiphase Flow 30(10): 1177-1192.

Hansen, A., Sinha, S., Bedeaux, D., Kjelstrup, S., Gjennestad, M.A. \& Vassvik, M. 2018. Relations between seepage velocities in immiscible, incompressible two-phase flow in porous media. Transport in Porous Media 125(3): 565-587.

Hisham, M.D., Rauf, A., Vieru, D. \& Awan, A.U. 2018. Analytical and semi-analytical solutions to flows of two immiscible Maxwell fluids between moving plates. Chinese Journal of Physics 56(6): 3020-3032.

Hristov, J. 2017. Transient space-fractional diffusion with a power-law super diffusivity: Approximate integral-balance approach. Fundamenta Informaticae 151(1-4): 371-388.

Hristov, J. 2019. Response functions in linear viscoelastic constitutive equations and related fractional operators. Mathematical Modelling of Natural Phenomena 14(3): 305.

Joseph, D.D. \& Renardy, Y.Y. 1995. Fundamentals of two-fluid dynamics. Journal of Fluid Mechanics 282: 405-405.

Kalogirou, A. \& Blyth, M.G. 2019. The role of soluble surfactants in the linear stability of two-layer flow in a channel. Journal of Fluid Mechanics 873: 18-48.

Khashi'Ie, N.S., Arifin, N.M., Pop, I. \& Nazar, R. 2020. Thermal Maranrgoni flow past a permeable stretching/shrinking sheet in a hybrid $\mathrm{Cu}-\mathrm{Al}_{2} \mathrm{O}_{3}$ /water nanofluid. Sains Malaysiana 49(1): 211-222.

Khan, Z., Islam, S., Shah, R.A. \& Khan, I. 2016. Flow and heat transfer of two immiscible fluids in double-layer optical fiber coating. Journal of Coatings Technology and Research 13(6): 1055-1063. 
Khuzhayorov, B., Auriault, J.L. \& Royer, P. 2000. Derivation of macroscopic filtration law for transient linear viscoelastic fluid flow in porous media. International Journal of Engineering Science 38(5): 487-504.

Kim, Y., Choi, H., Park, Y.G., Jang, J. \& Ha, M.Y. 2019. Numerical study on the immiscible two-phase flow in a nanochannel using a molecular-continuum hybrid method. Journal of Mechanical Science and Technology 33(9): 4291-4302.

Lake, L.W. 1989. Enhanced Oil Recovery. Englewood Cliffs, New Jersey: Prentice Hall.

Le Meur, H. 1997. Non-uniqueness and linear stability of the one-dimensional flow of multiple viscoelastic fluids. ESAIM: Mathematical Modelling and Numerical Analysis 31(2): 185-211.

Liu, J. \& Pan, D. 2019. Study on numerical solution of a variable order fractional differential equation based on symmetric algorithm. Sains Malaysiana 48(12): 2807-2815.

Lorenzo, C.F. \& Hartley, T.T. 1999. Generalized Functions for the Fractional Calculus. NASA. pp. 1-17.

Malek, J., Nečas, J. \& Růžička, M. 1993. On the non-Newtonian incompressible fluids. Mathematical Models and Methods in Applied Sciences 3(1): 35-63.

Nield, D.A. 2000. Modelling fluid flow and heat transfer in a saturated porous medium. Advances in Decision Sciences 4(2): 165-173.

Papaefthymiou, E.S. \& Papageorgiou, D.T. 2017. Nonlinear stability in three-layer channel flows. Journal of Fluid Mechanics 829(2017): 1-12.

Podlubny, I. 1999. Fractional Differential Equations, Vol. 198 of Mathematics in Science and Engineering. New York and London: Academic Press.

Rauf, A., Mahsud, Y. \& Siddique, I. 2019. Multi-layer flows of immiscible fractional Maxwell fluids in a cylindrical domain. Chinese Journal of Physics 67(2020): 265-282.

Russell, T.W.F. \& Charles, M.E. 1959. The effect of the less viscous liquid in the laminar flow of two immiscible liquids. The Canadian Journal of Chemical Engineering 37: 18-24.

Satpathi, D.K., Kumar, B.R. \& Chandra, P. 2003. Unsteadystate laminar flow of viscoelastic gel and air in a channel:
Application to mucus transport in a cough $\mathrm{m} \mathrm{a} \mathrm{c} \mathrm{h} \mathrm{i} \mathrm{n} \mathrm{e}$ simulating trachea. Mathematical and Computer Modelling 38(1-2): 63-75.

Vafai, K. \& Tien, C.L. 1981. Boundary and inertia effects on flow and heat transfer in porous media. International Journal of Heat and Mass Transfer 24(2): 195-203.

Ward, K., Zoueshtiagh, F. \& Narayanan, R. 2019. Faraday instability in double-interface fluid layers. Physical Review Fluids 4(4): 043903.

Xiao-Jun, X.J., Srivastava, H.M. \& Machado, J.T. 2016. A new fractional derivative without singular kernel. Thermal Sciences 20(2): 753-756.

Xue, C. \& Nie, J. 2008. Exact solutions of Rayleigh-Stokes problem for heated generalized Maxwell fluid in a porous half-space. Mathematical Problems in Engineering 1(2008): $1-10$.

Yih, C.S. 1967. Instability due to viscosity stratification. Journal of Fluid Mechanics 27(2): 337-352.

Abdul Rauf* \& Ali Majeed

Department of Computer Science and Engineering

Air University Multan Campus

Multan, 60000

Pakistan

Qammar Rubbab

Department of Mathematics

The Woman University Multan

Pakistan

Dumitru Vieru

Department of Theoretical Mechanics

Technical University of Iasi 700050

Romania

*Corresponding author; email: attari_ab092@yahoo.com

Received: 2 December 2019

Accepted: 31 May 2020 